\title{
Canonical HMG Protein
}

National Cancer Institute

\section{Source}

National Cancer Institute. Canonical HMG Protein. NCI Thesaurus. Code C16677.

The High Mobility Group (HMG) proteins were originally isolated from mammalian cells, named according to their electrophoretic mobility in polyacrylamide gels, and were arbitrarily classed as a specific type of non-histone proteins based on the observation that they are ubiquitous to mammalian cells, that they share certain physical properties, and that they are associated with isolated chromatin. Those mammalian proteins considered to be Canonical HMG proteins are now subdivided into 3 superfamilies: the HMGB (formerly HMG-1/-2) family, the HMGN (formerly HMG-14/-17) family, and the HMGA (formerly HMG-I/Y/C) family. Each HMG family has a characteristic functional sequence motif.

(www.informatics.jax.org/mgihome/nomen/genefamilies/hmgfamily.shtml) 